\title{
Correspondence
}

Irish Journal of Psychological Medicine, 39 (2022).

doi:10.1017/ipm.2019.48

\section{Breast implant illness and psychiatric implications}

Letter to the Editor,

Dear Sir or Madam,

I write with regard to the issue surrounding breast implant illness (BII) and its complications, many of which seem to be linked to mental health difficulties. It is important to note that BII is not an official medical diagnosis, but rather a term used by women with breast implants who self identify/describe an assortment of symptoms. Examples of these include fatigue, anxiety, depression, brain fog, sleep disturbances, alopecia, chest pain, photosensitivity, headaches, neurological issues, and so on and so forth (American Society for Aesthetic Plastic Surgery, 2019).

Lately, there seems to be an increasing number of women with these symptoms referred to medical teams and later for psychiatric input. Despite little or no robust epidemiological evidence associated between modern breast implants and BII, many women still experience such symptoms which appear to affect their quality of life and daily function. Self-reporting appears to have been influenced by the growth of social media as well as patient support groups, who aim to connect patients with similar concerns so that experiences are shared (Magnusson et al. 2019). Despite the lack of other scientific data, there has been some evidence supporting the induction of an autoimmune syndrome resembling fibromyalgia and chronic fatigue syndrome by silicone breast implants (Khoo et al. 2019). Nevertheless, it has been suggested that more prospective studies based on the development of depression in these patients are warranted.

Examining the recent media coverage of this issue, the British Broadcasting Corporation News (BBC News) had interviewed a patient who developed both physical and mental symptoms similar to those listed above, within weeks of having breast implants inserted. After a period of about 4 years, she elected to have them removed and within days, reported an improvement in symptoms (Collinson, 2019, BBC News). As a matter of fact, the BBC has reached out to surgical colleagues from the British Association of Aesthetic Plastic Surgeons. Contrary to the lack of evidence alluded to above, these professionals have indicated a need to warn women of BII symptoms during pre-operative consultation. It was also suggested that patients be informed that BII is currently poorly understood. Within the last 12 months, our service has come across two similar cases to the above. However unlike the case publicised by the BBC, onset of symptoms in our patients was more insidious, occurring around 7-8 years after breast implant insertion. Upon electing to have the implants removed, it took between 2-6 months for both physical and mental symptoms to abate.

Now it is important to understand that these are individual cases. There is no way to verify that alleviation of symptoms was directly related to removal of the implants. Such improvement may have been the result of other factors. It may even have been unrelated to the breast implants. Nevertheless, we need to acknowledge that it is the patients' difficulties that require management. During these times, we just have to rely on aspects of their history. Collateral from other healthcare professionals or family members may be useful. Ultimately, this will allow for treatment of psychiatric co-morbidities in such patients. Such actions are important due to the paradoxical nature of BII.

Breast implant surgery is thought to improve a patient's psychosocial and sexual well-being, selfesteem and also decrease depressive symptoms (Perez-San-Gregorio et al. 2016). However, if it appears that psychiatric and physical symptoms are exacerbated as a result of implants, then it may be viewed that such surgery poses the risk of being detrimental or at least unhelpful.

In summary, though BII is not an official medical diagnosis, we still have a duty of care to address and treat mental health issues in patients presenting with these difficulties. For now as Mental Health professionals, it is recommended that we liaise closely with our surgical and rheumatology colleagues regarding BII, should there be more causative evidence emerging in the future. Other issues that would need to be addressed later when we have a better understanding of the pathology of BII, would include treatment protocols and guidelines. At the moment, we would be expected to treat each entity, for example, depression, anxiety etc, as per standard guidelines.

Thank you.

Yours sincerely,

David A. Lachmansingh.

\section{Conflict of interest}

I have no conflicts of interest to disclose. 


\section{Ethical standards}

The authors assert that all procedures contributing to this work comply with the ethical standards of the relevant national and institutional committee on human experimentation with the Helsinki Declaration of 1975, as revised in 2008.

\section{Financial support}

This research received no specific grant from any funding agency, commercial or not-for-profit sectors.

\section{References}

American Society for Aesthetic Plastic Surgery (2019). Breast Implant Illness - Frequently Asked Questions/ Talking Points. (https://www.surgery.org/sites/default/ files/downloads/BII-Talking-Points-FINAL-1.15.19.pdf). Accessed 10 September 2019.

Collinson A (2019). Women "should be warned" of breast implant illness. BBC News. (https:/ / www.bbc.com/ news/health-49033715). Accessed 10 September 2019.
Khoo T, Proudman S, Limaye V (2019). Silicone breast implants and depression, fibromyalgia and chronic fatigue syndrome in a rheumatology clinic population. Clinical Rheumatology 38(5), 1271-1276.

Magnusson MR, Cooter RD, Rakhorst H, McGuire PA, Adams WP, Deva AK (2019). Breast Implant Illness: A Way Forward. Plastic and Reconstructive Surgery 143(3S), 74S-84S.

Pérez-San-Gregorio MA, Martín-Rodríguez A, AriasMoreno MJ, Rincón-Fernández ME, Ortega-Martínez JI (2016). Self-reported psychological development in cosmetic breast surgery patients. Medicine (Baltimore) 95(49), e5620.

David ANTOINe LaChMANSINGH

Department of Psychiatry, St. Stephen's Hospital, Glanmire, Co. Cork, Ireland

Department of Medicine, University College Cork, Brookfield Health Sciences Complex, College Road, Cork, Ireland Department of Pharmacology and Therapeutics, University College Cork, Western Gate Building, Western Road, Cork, Ireland (Emails: davidlachmansingh@yahoo.com, 103140679@umail.ucc.ie) 